\title{
Génétique et évaluation du potentiel industriel des souches
}

\author{
P Renault, C Delorme, JJ Godon, SD Ehrlich
}

Laboratoire de génétique microbienne, INRA, 78352 Jouy-en-Josas Cedex, France

\begin{abstract}
Résumé - L'ensemble des propriétés d'une souche qui confère au produit ses qualités et dirige le procédé technologique est en général le résultat de l'expression d'un grand nombre de gènes et de la régulation de chacun d'entre eux. Cette complexité entraîne l'absence de méthode génétique directe de mesure du potentiel d'une souche. L'analyse biochimique des produits ne permet pas non plus d'apprécier de manière fiable la qualité d'un produit. II en résulte que seule une fabrication suivie d'une dégustation permet de définir correctement les qualités d'une souche. Cette méthode est lourde et ne peut donc s'appliquer qu'à un nombre limité de souches.

Le développement très rapide des techniques de biologie moléculaire peut permettre d'alléger ce travail. En effet, si actuellement seul un petit nombre de caractères peut être détecté par ces méthodes, la taxonomie moléculaire permet de classer les différents clones d'une espèce en sousespèces, groupes et sous-groupes à l'intérieur desquels les souches sont génétiquement très proches, et dont les propriétés sont voisines. L'obtention d'une classification phylogénique des clones d'une espèce pourrait ainsi permettre de choisir rationnellement les souches à tester.

L'étude du polymorphisme par RFLP, PFGE, PCR, et séquence nucléotidique chez les lactocoques a donné un aperçu de la diversité génétique des souches isolées en laiterie et dans d'autres milieux naturels. Les deux sous-espèces $L$ lactis subsp lactis et $L$ lactis subsp cremoris, que des tests phénotypiques ne permettent pas de discriminer avec certitude, se sont révélées relativement éloignées sur le plan génétique. Des sondes ont été développées pour les distinguer sans ambiguinté. À l'intérieur de ces sous-espèces, un certain nombre de groupes peuvent être differenciés et la séquence nucléotidique de plusieurs clones a permis de mesurer leur éloignement génétique. L'utilisation systématique de ces méthodes, suivie d'un traitement statistique des données, et leur intégration par des logiciels adaptés, devraient permettre d'évaluer la richesse génétique des espèces et de réaliser des approches raisonnées d'étude et de sélection des souches
\end{abstract}

Lactococcus lactis / typage / phylogénie / sonde / diversité

Summary - Genetics and evaluation of the industrial potential of lactic acid bacteria. The technological properties of a strain which influence the manufacturing process and the quality of the product is the result of the expression and regulation of a great number of genes. Such complexity impedes the development of direct genetic measures of the industrial potential of strains. Moreover, the biochemical analysis of products is not yet reliable. The only available method to evaluate the quality of a strain is thus to use it for small-scale production, which is time-consuming and thus limited to a restricted number of strains. The rapid development of molecular biology might alleviate this problem. Although a very limited number of characters can be detected by these methods, molecular taxonomy leads to the classification of strains into genetically related species, subspecies, groups and subgroups which share a number of properties. The construction of a phylogenic tree with different clones of a species would allow a more rational choice of the strains to be tested. The study of 
polymorphism by RFLP, PFGE, PCR and nucleotide sequences of strains from the genus Lactococcus gives a first view of the genetic diversity of lactococcal strains isolated from dairy products and from some other biotopes. The 2 subspecies $L$ lactis subsp lactis and $L$ lactis subsp cremoris which can not be properly discriminated by phenotypical tests are not closely related genetically. Their nucleotide sequences diverge by 15 to $20 \%$. Two subspecies probes have been developed to distinguish them unambiguously. Within a subspecies, some groups can be differentiated and the nucleotide sequence of some clones allowed a direct measure of their relatedness. The maximum divergence detected was $3 \%$. The systematic use of these methods followed by statistical analysis of the data would improve our knowledge on the genetic potential in a species and enable a rational approach for the selection of the strains to be established.

\section{Lactococcus lactis / phylogeny / probe / genetic diversity / strain}

\section{INTRODUCTION}

Lactococcus lactis subsp lactis et subsp cremoris sont utilisées couramment dans la fabrication de fromages et d'autres produits laitiers fermentés. Depuis des dizaines, voire des centaines d'années, un nombre important de souches ont pu s'adapter à cette utilisation et évoluer. Elles peuvent différer par un certain nombre de propriétés importantes pour l'industrie, telles que leur vitesse d'acidification, leurs activités protéolytiques et aromatiques et leur sensibilité aux phages.

La conception de levains mieux définis permettra dans un futur proche d'utiliser des souches améliorées par les techniques de la biologie moléculaire. Les souches utilisées à cet effet devraient avoir des propriétés répondant aux exigences d'une fabrication fiable et de produits de qualité. Parmi les nombreuses souches collectées jusqu'à présent, il faudra opérer un choix selon leurs caractéristiques industrielles mais aussi selon des critères génétiques. En effet, il peut être important de conserver le maximum de diversité génétique au sein de l'ensemble des souches qui seront utilisées, afin de satisfaire aux exigences de fabrication de différents produits et de conserver la possibilité d'élaborer de nouveaux procédés.
Dans cette optique, il est donc indispensable de classer les souches d'une collection selon leur parenté, ce qui permettra de définir un certain nombre de familles, dont seuls quelques représentants seront étudiés.

Actuellement, le classement d'une souche en espèce et éventuellement sous-espèce se fait principalement suivant des caractères phénotypiques (test de croissance en différentes conditions, utilisation de substrats, présence de quelques activités enzymatiques et éventuellement composition des parois et GC\%) (Garvie et Farrow,1982; Garvie, 1984 ; Schleifer et Kilper-Balz, 1987). Cependant, ces caractères ne permettent pas toujours une classification correcte des souches. Pour pallier à cette déficience, diverses techniques moléculaires ont été mises au point. Les analyses par "restriction fragment length polymorphism" (RFLP) des gènes codant pour les ARN ribosomiques (Kohler et al, 1991; Rodrigues et al, 1991) ou l'emploi de sondes oligonucléotides spécifiques de régions hypervariables des ARN $23 S$ et 16S (Betzl et al, 1990; Salama et al, 1991; Klijn et al, 1991) peuvent être utilisés pour classer les souches en espèces et même parfois en sous-espèces. Ces techniques peuvent être complétées par des analyses de profils plasmidiques et 
de restriction de I'ADN chromosomique en champ pulsé (PFGE) (Tanskanen et al, 1990; Lebourgeois et al, 1991) et éventuellement l'analyse de séquences générant du polymorphisme comme les IS (Schaffer et al, 1991).

II est ainsi possible de classer correctement les souches et de distinguer les clones les uns des autres, mais non d'évaluer l'éloignement génétique de ces souches. La variabilité génétique au sein d'une population peut cependant fortement différer en fonction de la manière dont les souches sont disséminées et ont été sélectionnées (naturellement ou par l'homme) et du temps depuis lequel s'opère la diversification. La divergence entre différentes souches peut être mesurée directement par la différence entre 2 séquences homologues; celle-ci augmentera avec la divergence des souches. La richesse génétique dans une population (par exemple dans une collection) dépend principalement de la divergence maximale entre les souches et de la manière dont elles ont divergé. Par exemple, une population se répartissant selon l'arbre de la figure $1 \mathrm{~A}$ est composée de clones relativement différents les uns des autres, et pourra être considérée comme riche et diversifiée. Au contraire, la population représentée sur la figure $1 \mathrm{~B}$ n'est composée que de 2 familles peu différentes l'une de l'autre.

Dans cet article, nous utiliserons les différentes méthodes de la biologie moléculaire pour mieux caractériser la diversité génétique au sein de l'espèce $L$ lactis. En particulier nous quantifierons la divergence de certaines souches types par la comparaison de la séquence nucléotidique de certains gènes, et établirons les bases d'une classification phylogénique. Ces résultats permettent d'espérer des progrès importants dans la valorisation de ressources génétiques jusqu'alors sousexploitées.
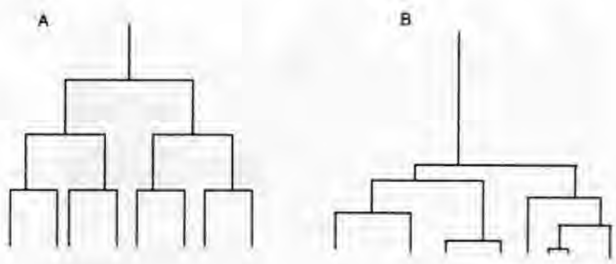

Fig 1. Exemple d'arbre phylogénique où les souches sont classées en fonction du degré de divergence de 2 séquences homologues. Le point où se situe un embranchement indique le pourcentage de divergence entre les souches présentes de part et d'autre. Cette divergence est le reflet du temps et de la vitesse de divergence des séquences comparées.

\section{ANALYSE PAR RFLP}

L'analyse par RFLP est une technique simple. L'ADN chromosomique de souches bactériennes est clivé par des enzymes de restriction; les fragments ainsi produits sont séparés par électrophorèse, puis transférés sur un filtre. Les fragments homologues à une sonde ou à une série de sondes marquées sont alors visualisés par hybridation. Les souches d'une même espèce ont en général des profils de restriction semblables. Les différences de profils dues à l'apparition ou à la disparition de certains sites dans des régions homologues aux sondes dépendent de leur degré de divergence. Un exemple représentatif de ce type d'analyse est présenté sur la figure 2 où la sonde utilisée est un fragment de $20 \mathrm{~kb}$ contenant l'opéron de biosynthèse des acides aminés branchés (Godon et al, 1992a). Les profils de restrictions des différentes souches de $L$ lactis subsp lactis sont très semblables (excepté celui de la souche NCDO763). De même, les profils obtenus avec l'ADN de différentes souches de $L$ lactis subsp cremoris sont homogènes, mais ils diffèrent totalement de 


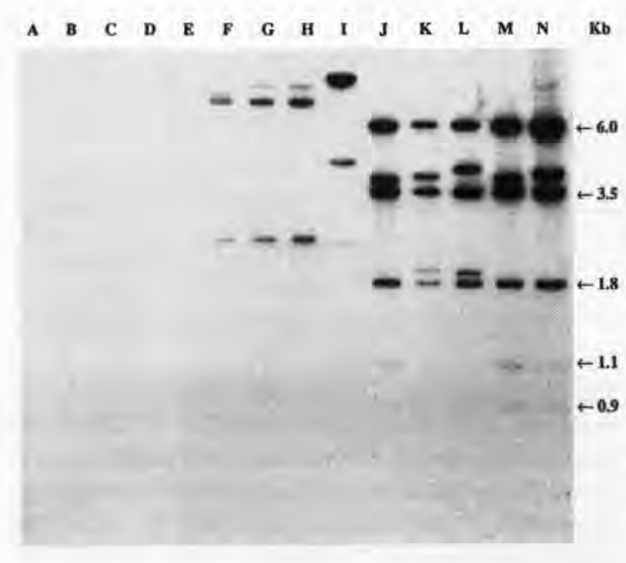

Fig 2. Analyse par hybridation de différentes souches du genre Lactococcus subsp. L'ADN chromosomique a été coupé par EcoRl et hybridé à basse stringence avec le plasmide pIL500, qui contient un fragment de $18,5 \mathrm{~kb}$ codant pour l'opéron ilv de L lactis subsp lactis (Godon et al, 1992a, b). La taille du marqueur est donnée en kb. Puits : A) Bacillus subtilis 168; B) E faecalis CG110; C) S thermophilus CNRZ302; D) L raffinolactis NCDO0617; E) L garviae NCDO2155; F) L lactis subsp cremoris Z357; G) L lactis subsp cremoris NCDO607; H) L lactis subsp cremoris AM2; I) $L$ lactis subsp lactis NCDO763; J) $L$ lactis subsp lactis biovar diacetylactis Z430; K) L lactis subsp lactis Z148; L) L lactis subsp lactis IL1403; M) L lactis subsp lactis NCDO2125; N) L lactis subsp lactis NCDO2118.

ceux de la sous-espèce lactis. Ce type de résultat a également été obtenu avec d'autres sondes, telles que la thioredoxine réductase (Renault, 1990), la X-prolyl dipeptidyl aminopeptidase (Nardi et al, 1991), l'opéron histidine (Delorme et al, 1992), l'opéron tryptophane (Bardowski et al, 1992), et l'aminopeptidase C (ChapotChartier et al, 1993). Les 2 sous-espèces sont donc distinctes génétiquement et forment des groupes homogènes (mis à part le cas particulier de la souche NCDO763, discuté ci-dessous), (Godon et al, 1992b).

\section{ANALYSE PAR HYBRIDATION SUR COLONIES}

L'analyse par RFLP, bien que conceptuellement simple, est techniquement trop lourde à utiliser pour effectuer un tri systématique d'une grande collection. Dans ce cas, ainsi que pour des analyses de routine, l'utilisation de techniques comme l'hybridation de colonies sur filtre est plus appropriée. Une centaine de souches peuvent être déposées sur un filtre qui, après un traitement adéquat, est hybridé avec une sonde. Les colonies dont I'ADN contient une séquence homologue à la sonde se distinguent par un signal intense. Cette technique permet de repérer des souches pour lesquelles une sonde spécifique de chaque groupe à différencier est disponible. Nous disposons de 2 sondes spécifiques aux 2 sous-espèces lactis et cremoris (fig 3 ). Leur utilisation permet de classer sans ambiguïté une souche de $L$ lactis dans l'un des groupes lactis ou cremoris (fig 4).

Les classifications obtenues par méthodes phénotypiques (production d'ammoniaque à partir d'arginine, croissance à $\mathrm{pH} 9,2$, avec $4 \% \mathrm{NaCl}$ ou à $42{ }^{\circ} \mathrm{C}$ ) et génétiques concordent pour une majorité de souches (plus de 95\%) prises dans un échantillon de notre collection. Cependant, un certain nombre de souches présentant les caractères phénotypiques de l'une des sous-espèces font partie génétiquement de l'autre (tableau I). En particulier, toutes les souches affiliées à la NCDO712 (NCDO763, ML3, C2, ...) qui sont utilisées comme souches modèles par un grand nombre de laboratoires, font partie du groupe cremoris. Le rattachement de ces souches au groupe cremoris est en accord avec la comparaison des séquences du gène pepXP (Mayo et al, 1991; Nardi et al, 1991), des données basées sur la réassociation ADN/ADN (Jarvis et Jarvis, 1981) 


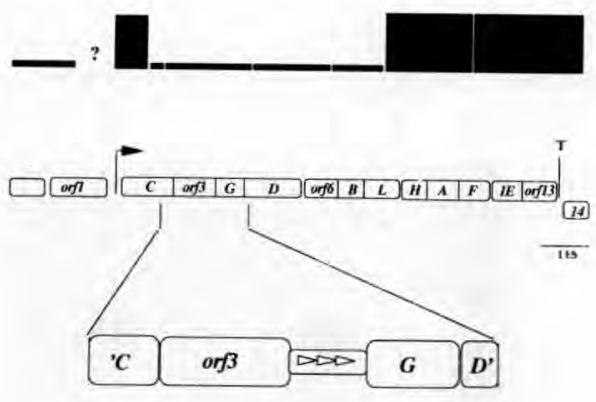

Fig 3. Organisation et homologie de l'opéron histidine chez $L$ lactis subsp lactis et subsp cremoris. Les différentes sondes de $L$ lactis subsp lactis NCDO2118 utilisées pour des hybridations contre l'ADN de différentes souches de $L$ lactis subsp cremoris ont été indiquées par des rectangles noirs. Un rectangle épais indique l'obtention d'un signal d'hybridation intense après une hybridation en présence de $50 \%$ de formamide. Un rectangle étroit indique l'obtention d'un signal détectable après hybridation en présence de $25 \%$ de formamide, mais faible à absent à $50 \%$. La séquence agrandie correspond à la partie de l'opéron his clonée chez $L$ lactis NCDO2118 et NCDO763 utilisée comme sonde sur la figure 4 et dont la séquence nucléotidique a été déterminée. Les 3 triangles entre orf3 et hisG représentent des séquences non codantes, répétées un nombre variable de fois suivant les souches (1 à 4) et présentes uniquement dans le groupe cremoris.

et les séquences des ARN ribosomiques (Salama et al, 1991). L'utilisation de ces méthodes a permis de plus, de montrer que les bactéries de la sous-espèce $L$ lactis subsp hordniae font partie du groupe lactis.

Cette nouvelle classification est en accord avec le groupement des souches selon leur sensibilité aux bactériophages. Ainsi, après le reclassement de certaines souches à l'aide de nos sondes, les différents groupes de bactéries et de phages définis dans des travaux précédents (Cho-
Tableau I. Souches dont la classification doit être révisée.

L lactis subsp lactis ex-cremoris
\begin{tabular}{ll} 
CNRZ193 & IL927 \\
CRNZ342 & CNRZ337 \\
IL180 & IL921 \\
IL994 & \\
\multicolumn{2}{l}{} \\
L lactis subsp cremoris ex-lactis \\
NCDO763 & IL969 \\
8M & CNRZ306 \\
ML3 & SK1
\end{tabular}

pin et al, 1976) sont homogènes. En effet, les phages d'un groupe n'attaquent que des bactéries de l'une des sous-espèces soit lactis soit cremoris; la seule exception est une souche du groupe cremoris, qui est sensible à 2 groupes de phages dont un est spécifique des lactis.

D'un point de vue taxonomique, le critère d'hybridation qui compare directement les informations génétiques est un test plus fiable, et en cas de divergence avec les critères phénotypiques doit prévaloir.

\section{MESURE DE LA DIVERGENCE ENTRE LES GROUPES LACTIS ET CREMORIS}

Les méthodes utilisées précédemment permettent de bien différencier les sousespèces, mais ne mesurent pas précisément leur divergence. En première approximation, les données obtenues par hybridation à différentes stringences avec les différentes sondes indiquent que les séquences nucléotidiques des lactis et des cremoris divergent de 15 à $30 \%$. Cette divergence est relativement élevée (comparable à la divergence entre Salmonella et Escherichia (Ochman et Wilson, 1987)). 

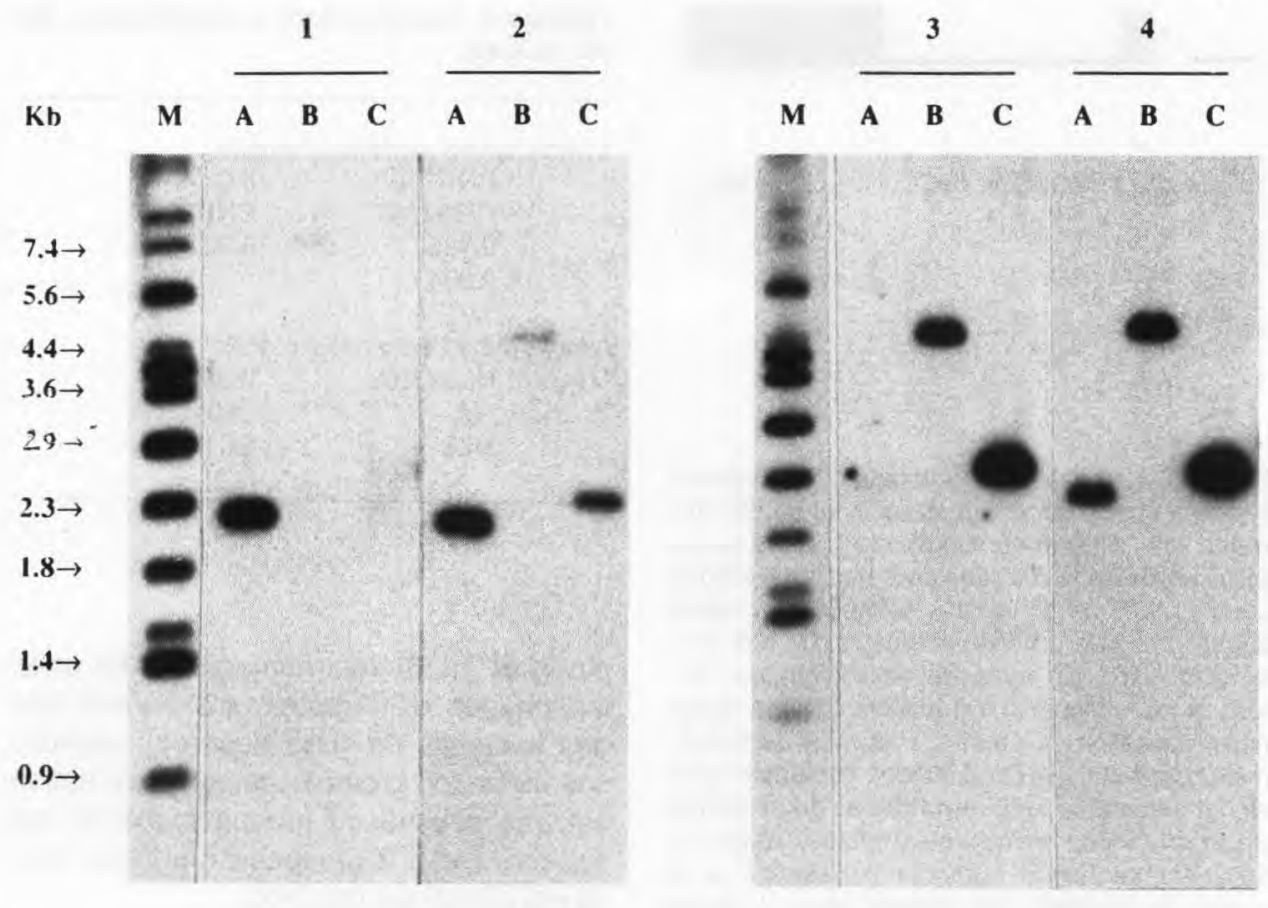

Fig 4. Analyse par hybridation de 3 souches de $L$ lactis avec 2 sondes à forte et à faible stringence (50 et $25 \%$ de formamide). Puits : A) L lactis subsp lactis NCDO2118; B) L lactis subsp cremoris AM2; C) L lactis subsp lactis NCDO763. Filtres 1 et 2 : sonde constituée d'une partie de l'opéron his de $L$ lactis subsp lactis NCDO2118 à forte (filtre 1) et à faible stringence (filtre 2). Filtres 3 et 4 : sonde constituée du fragment homologue à celui utilisé pour les filtres 1 et 2 mais isolé de $L$ lactis subsp cremoris (ex-lactis) NCDO763 à forte (filtre 3) et à faible stringence (filtre 4).

Une mesure plus détaillée de la divergence a été réalisée en comparant des séquences nucléotidiques homoiogues d'environ $2,3 \mathrm{~kb}$ d'une souche de chaque groupe (Delorme, 1992). La région séquencée comprend la fin du gène hisC, un gène homologue à une histidyl ARNt synthétase (orf3), le gène hisG et le début du gène hisD (fig 3 ). Le fragment de la souche NCDO763 est plus long de 201 paires de bases, à cause de l'insertion d'une séquence d'ADN entre les gènes orf3 et hisG. L'homologie moyenne entre ces 2 fragments est de $73 \%$ mais la distribution des changements n'est pas homogène. En effet, cette homologie est supérieure à $75 \%$ et $80 \%$ dans les 480 premières et les 740 dernières paires de bases, ce qui correspond à la différence détectée par hybridation avec différentes sondes (voir plus haut). En revanche, cette homologie n'est que de 25 à $60 \%$ dans la partie centrale qui de plus possède plusieurs particularités : son contenu en 
bases GC est très élevé ( $54 \%$ contre $36 \%$ en moyenne sur le chromosome), le biais de codon est atypique par rapport à celui des lactocoques et une séquence supplémentaire est insérée entre 2 gènes. L'ensemble de ces données suggère que la zone centrale (ainsi que probablement d'autres régions dans les séquences environnantes) provient d'un transfert de matériel génétique à partir d'un microorganisme relativement distant (GC\%, homologie, biais de codon). Ce transfert aurait suivi la séparation des deux sousespèces.

La mesure de la divergence par comparaison de séquences homologues n'est en fait valable qu'en l'absence de transfert de matériel d'une souche à l'autre. Dans le cas présent, seules les parties "endogènes" doivent être prises en compte. Compte tenu de ces réserves, la valeur de 15 à $25 \%$ de divergence des séquences conservées suggère que l'émergence des 2 sous-espèces est antérieure au développement des techniques laitières (plusieurs millions voire dizaines de millions d'années). Ces souches se seraient donc adaptées au milieu «lait» indépendamment.

\section{DIVERSITÉ GÉNÉTIQUE AU SEIN DE CHAQUE SOUS-ESPĖCE}

Les données exposées ci-dessus montrent clairement la division de l'espèce $L$ lactis en deux sous-espèces, fortement divergentes. Au sein de chacune de ces sousespèces existe une diversité génétique montrée par les analyses par RFLP, mais surtout par électrophorèse en champ pulsé. Cependant, aucune de ces techniques ne peut permettre d'estimer la divergence, et donc la richesse génétique au sein de chacune de ces sous-espèces.
Des séquences nucléotidiques homologues d'un certain nombre de souches ont été déterminées dans ce but (fig 5 ).

Le groupe lactis est représenté par $L$ lactis subsp lactis IL1403 (isolé du lait) et NCDO2118 (isolé de pois congelés) et $L$ lactis subsp hordniae NCDO2181 (isolé d'insecte). L'analyse de séquence de 1,5 $\mathrm{kb}$ interne à l'opéron histidine montre que $L$ lactis subsp hordniae diverge légèrement plus des 2 autres lactocoques $(2,3 \%)$ que ceux-ci entre eux $(1,3 \%)$.

Toutes les souches du groupe cremoris ont été isolées de produits laitiers. La souche NCDO763 diverge de $3,5 \%$ des souches IL182 et AM2, alors que celles-ci sont très proches entre elles (moins de $0,6 \%$ de divergence). L'analyse d'une séquence plus courte (200 à 300 nucléotides) et à fort polymorphisme contenant les séquences répétées entre les gènes orf3 et hisG chez 10 souches suggère que le groupe cremoris peut être divisé en 2 sous-groupes. À l'intérieur de ces groupes, aucune des souches n'est exactement pareille à l'autre, mais elles diffèrent entre elles de moins de $1 \%$.

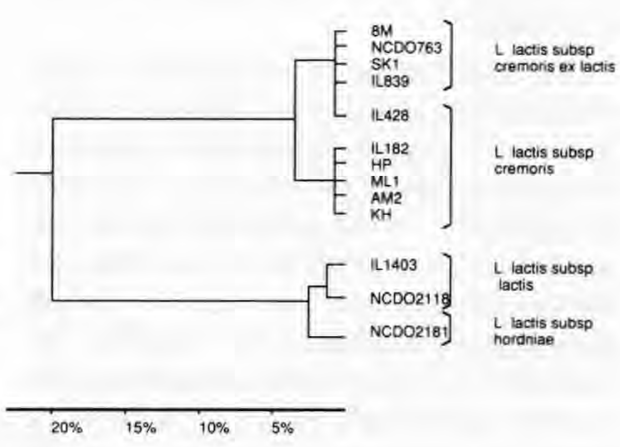

Fig 5. Arbre phylogénique obtenu d'après des comparaisons de séquences. En abscisses est indiqué le pourcentage de divergence des séquences des souches présentes de part et d'autre des embranchements. 


\section{CONCLUSION}

Le recensement de la diversité génétique chez les lactocoques présenté dans cet article n'est pas exhaustif, et en particulier l'échantillonnage de la sous-espèce $L$ lactis subsp lactis est restreint. D'un point de vue génétique, l'espèce $L$ lactis se différencie clairement des autres espèces de ce genre ( $L$ garviae, $L$ plantarum, $L$ raffinolactis), car aucune des sondes chromosomiques utilisées ne permet de révéler un signal significatif contre leur ADN chromosomique.

Au sein de l'espèce $L$ lactis, 2 sousensembles se distinguent nettement par hybridation. Le premier regroupe majoritairement des souches de phénotype cremoris. Cette sous-espèce contient 2 sousgroupes; le plus important numériquement contient principalement des souches de phénotype cremoris, alors que le second contient des souches généralement classées jusqu'ici comme des lactis. La diversité génétique au sein de chaque groupe semble relativement faible (moins de $1 \%$ de divergence dans des zones à fort polymorphisme). II est intéressant de constater que toutes les souches de la sous-espèce cremoris proviennent du milieu lait et de ses derivés.

Les souches de la sous-espèce lactis peuvent être isolées de différents milieux comme les produits laitiers, les végétaux, le système digestif des insectes, l'industrie du poisson... II est probable qu'elle soit composée de nombreux clones dont les séquences divergent de 1 à $3 \%$. Le séquençage systématique de certaines séquences très polymorphes présentes dans l'opéron leucine permettrait sans doute de mettre en évidence des groupes homogènes au sein de cette sous-¿spèce, et en particulier, de savoir si les souches isolées du lait sont génétiquement plus proches les unes des autres qu'elles ne le sont de souches isolées d'autres milieux.
Toutefois, le séquençage systématique d'une région même restreinte du chromosome pour un très grand nombre de souches pose des problèmes matériels qui rendent nécessaire la mise au point de techniques d'analyse simplifiées. Son application à un échantillonnage représentatif, devrait permettre de définir des groupes et de concevoir des sondes spécifiques pour chacun d'entre eux. Ces sondes seraient alors utilisées pour classer l'ensemble des souches collectées. Le classement phylogénique, complété avec des données sur la sensibilité aux phages et l'aptitude des souches en fromagerie, permettrait de dégager les caractéristiques intrinsèques aux familles de souches. L'avantage d'un tel classement vient du fait que les propriétés chromosomiques des souches d'une même famille seront en général très voisines; il sera ainsi possible de rechercher rapidement soit des variants au sein d'une famille, soit au contraire des souches répondant à un besoin nouveau au sein d'un échantillonnage restreint.

\section{RÉFÉRENCES}

Bardowski J, Ehrlich SD, Chopin A (1992) Tryptophan biosynthesis genes in Lactococcus lactis subsp lactis. J Bacteriol 174, 6563-6570

Betzl D, Ludwig W, Schleifer K (1990) Identification of lactococci and enterococci by colony hybridization with 23S rRNA-targeted oligonucleotides probes. Appl Environ Microbiol 56, 2927-2929

Chapot-Chartier, MP, Nardi M, Chopin MC, Chopin A, Gripon JC (1993) Cloning and sequencing of pepC, a cysteine aminopeptidase gene from Lactococcus lactis subsp cremoris AM2. Appl Environ Microbiol 59, 330-333

Chopin, MC, Chopin A, Roux C (1976) Definition of bacteriophage groups according to their Iytic action on mesophilic lactic streptococci. Appl Environ Microbiol 32, 741-746

Delorme C,Ehrlich SD, Renault P (1992) Histidine biosynthesis genes in Lactococcus lactis subsp lactis. J Bacteriol 174, 6571-6579 
Delorme C (1992) Polymorphisme de l'opéron histidine chez les Lactocoques. Thèse univ Paris VII

Garvie, El (1984) Taxonomy and identification of bacteria important in cheese and fermented dairy product. In: Advances in the microbiology and biochemistry of cheese and fermented milk (Davies FL, Law BA, eds). Elsevier Applied Science Publisher, London

Garvie El, Farrow JAE (1982) Streptococcus lactis subsp cremoris (Orla-Jensen) comb nov and Streptococcus lactis subsp diacetilactis (Matuszewski et af) nom rev comb nov. Int J Syst Bacteriol 32, 453-455

Godon JJ, Chopin MC, Ehrlich SD (1992a) Branched chain amino acid biosynthesis genes in Lactococcus lactis subsp lactis. J Bacteriol $174,6580-6589$

Godon JJ, Delorme C, Ehrlich SD, Renault P (1992b) Divergence of genomic sequences between Lactococcus lactis subsp lactis and Lactococcus lactis subsp cremoris. Appl Environ Microbiol 58, 4045-4047.

Jarvis AW, Jarvis BDW (1981) Deoxyribonucleic acid homology among lactic streptococci. Appl Environ Microbiol 41, 77-83

Klijn N, Weerkamp AH, de Vos WM (1991) Identification of mesophilic lactic acid bacteria by using polymerase chain reaction-amplified variable regions of $16 \mathrm{~S}$ rRNA and specific DNA probes. Appl Environ Microbiol 57. 3390-3393

Kohler G, Ludwig W, Schleifer K (1991) Differentiation of lactococci by rRNA gene restriction analysis. FEMS Microbiol Lett 84, 307-312

Lebourgeois P, Mata M, Ritzenthaler P (1991) Pulsed-fied gel electrophoresis as a tool for studying the phylogeny and genetic history of lactococcal strains. In: Steptococcal genetics (Dunny GM, Cleary PP, McKay LL, eds). American Society for Microbiology, Washington DC

Mayo B, Kok J, Venema K, Bockelmann W, Teuber M, Reinke H, Venema G (1991) Molecu- lar cloning and sequence analysis of the $X$ prolyl dipeptidyl aminopeptidase gene from Lactococcus lactis subsp cremoris. Appl Environ Microbiol 57, 38-44

Nardi M, Chopin MC, Chopin A, Cals MM, Gripon JC (1991) Cloning and DNA sequence analysis of an X-prolyl dipeptidyl aminopeptidase gene from Lactococcus lactis subsp lactis NCDO763. Appl Environ Microbiol 57, 4550

Ochman H, Wilson AC (1987) Evolutionary history of enteric bacteria. In: Escherichia coli and Salmonella typhimurium: Cellular and molecular biology (Neidhardt FC, ed). American Society for Microbiology, Washington DC

Renault P (1990) Malolactic fermentation: genetics and genetic engineering. In: 6th international symposium on genetics of industrial microorganisms (Heslot H, Davies J, Florent J, Bobichon L, Durant G, Penasse L, eds). Société Francaise de Microbiologie, Paris

Rodrigues U, Aguirre M, Facklam R, Collins M (1991) Specific and intraspecific molecular typing of lactococci based on polymorphism of DNA encoding rRNA. J Appl Bacteriol 71, 509-516

Salama M, Sandine W, Giovannoni S (1991) Development and application of oligonucleotide probes for identification of Lactococcus lactis subsp cremoris. Appl Environ Microbiol 57, 1313-1318

Schaffer A, Jahns A, Feis A, Teuber M (1991) Distribution of the ISS1 and IS904 in lactococci. FEMS Microbiol Lett 80, 311-318

Schleifer KH, Kilper-Balz R (1987) Molecular and chemotaxonomic approaches to the classification of Streptococci, Enterococci and Lactococci: a review. Syst Appl Microbiol 10, 1-19

Tanskanen E, Tulloch D, Hillier A, Davidson B (1990) Pulsed-field gel elctrophoresis of Smal digest of lactococcal genomic DNA, a novel method of strain identification. Appl Environ Microbiol 56, 3105-3111 